\title{
LAPLACE SERIES AND SETS OF LOGARITHMIC CAPACITY ZERO
}

WALTER RUDIN

In the principal theorems of [2] and [3] sets of capacity zero ("capacity" will mean "logarithmic capacity" in this note) appear in the hypotheses. More specifically, conditions like finiteness of generalized Laplacians or of Poisson sums are assumed to hold everywhere except possibly on a closed set of capacity zero; these sets play the role of sets of uniqueness. The present note will show that the conclusions of these theorems become false if the sets of capacity zero are replaced by any larger class of closed sets.

We begin with the following known theorem [6, p. 84].

Theorem A. If $e$ is a plane compact set of positive capacity, then there exists a positive measure $\mu$, concentrated on $e$, such that the potential

$$
v(z)=\int_{0} \log |\zeta-z| d \mu(\zeta)
$$

is continuous in the whole plane.

By a measure we mean a finite real-valued (not necessarily nonnegative) completely additive set function defined for all Borel sets.

Since $v$ is subharmonic in the plane and harmonic in the complement of $e$, it follows that Theorems I and II of [2] become false if exceptional sets of positive capacity are admitted.

As $z \rightarrow \infty, v(z)$ differs from $\mu(e) \cdot \log |z|$ by a bounded harmonic function. To construct a nonconstant function $v$ of the form (1) which is also regular at infinity, we take two disjoint closed subsets $e_{1}$ and $e_{2}$ of $e$, both of positive capacity, and then choose positive measures $\mu_{i}$ concentrated on $e_{i}(i=1,2)$ such that the associated potentials $v_{i}$ are continuous in the plane, and such that $\mu_{1}\left(e_{1}\right)=\mu_{2}\left(e_{2}\right)$. If $\mu=\mu_{1}-\mu_{2}$, then $\mu(e)=0$, and

$$
v(z)=\int_{e} \log |\zeta-z| d \mu(\zeta)=v_{1}(z)-v_{2}(z)
$$

has the desired properties.

We now turn to series of spherical surface harmonics $Y_{n}(P)$, where $P$ denotes a point of the surface $S$ of the unit sphere in Euclidean 3 -space. The notation and nomenclature will be the same as in [3],

Received by the editors February 9, 1955. 
and for the sake of brevity we shall not repeat it all.

Theorem B. Let $E$ be a closed set of positive capacity on $S$. There exists a series $\sum_{0}^{\infty} Y_{n}(P)$ with the following properties:

(i) $\sum_{1}^{\infty} Y_{n}(P)$ does not vanish identically, but for every $P$ in $S-E$ the series is $(C, 1)$-summable to 0 and Riemann-summable to 0.

(ii) $-\sum_{1}^{\infty} Y_{n}(P) / n(n+1)$ is the Laplace series of a continuous function $F$ on $S$.

(iii) If, in addition, $E$ is such that every diameter of $S$ has either both or none of its end points in $E$, then, for every $P$ in $S-E$ and for every $k>1 / 2, \sum_{0}^{\infty} Y_{n}(P)$ is $(C, k)$-summable to 0 .

Consequently closed sets $Z$ of positive capacity cannot be admitted in the hypotheses of Theorems 2.6 and 2.7 of [3].

Proof. Let $N$ be a point in $S-E$, and project $S$ stereographically on the plane through the center of $S$, with $N$ as center of projection, taking $E$ into a plane set $e$ of positive capacity. If $P$ and $Q$ on $S$ correspond to $z$ and $\zeta$ in the plane and if $V(P)=v(z) / 2 \pi$, where $v(z)$ is given by (2), then $V$ is continuous on $S$ and harmonic in $S-E$; also, if $P Q$ denotes the shorter great circle arc on $S$ between $P$ and $Q$, we have

$$
K(P, Q) \equiv \log \left\{\frac{\sin (N P / 2) \sin (N Q / 2)}{\sin (P Q / 2)}\right\}=-\log |\zeta-z|,
$$

as is easily verified by considering the singularities of the two functions, and their harmonic character.

Let $\lambda$ be the measure (concentrated on $E$ ) obtained by transplanting $\mu$ to $S$ by means of our projection. Then

$$
V(P)=-\frac{1}{2 \pi} \int_{E} K(P, Q) d \lambda(Q)
$$

Recalling that $\lambda(E)=0$, (3) shows that $V(P)$ differs from

$$
F(P)=\frac{1}{2 \pi} \int_{E} \log \sin (P Q / 2) d \lambda(Q)
$$

by a constant (we draw the reader's attention to the analogy between (5) and the function $\Omega f$ defined in [3, p. 293]). The function $F$ has the following properties:

(6) $F$ is continuous and not constant on $S$;

(7) $F$ is harmonic in $S-E$;

(8) $\iint_{S} F(P) d P=0$, since $\lambda(E)=0$.

Now define $Y_{n}(P)(n \geqq 1)$ by the requirement that 


$$
-\sum_{1}^{\infty} Y_{n}(P) / n(n+1)
$$

be the Laplace series of $F$; this is possible by (8) and implies

$$
-\frac{Y_{n}(P)}{n(n+1)}=\frac{2 n+1}{4 \pi} \iint_{S} F(Q) P_{n}(\cos P Q) d Q \quad(P \text { on } S, n \geqq 1)
$$

where $P_{n}$ is the Legendre polynomial of degree $n$. Substituting (5) into (9) and reversing the order of integration, we obtain (compare $[3$, Theorem 5.2])

$$
Y_{n}(P)=\frac{2 n+1}{4 \pi} \int_{E} P_{n}(\cos P Q) d \lambda(Q) \quad(n \geqq 0) .
$$

In other words, $\sum_{0}^{\infty} Y_{n}(P)$ is the Laplace-Stieltjes series of the measure $\lambda$. Note that $Y_{0}$ is defined by (10), and that $Y_{0}=0$.

By $(7), \sum_{0}^{\infty} Y_{n}(P)$ is Riemann-summable to 0 in $S-E$.

Let $s_{n}^{(k)}(\theta)$ be the $n$th Cesàro mean of order $k$ of the series

$$
\sum_{n=0}^{\infty}(2 n+1) P_{n}(\cos \theta) .
$$

For $1 / 2<k \leqq 1$ and $0<\theta<\pi$, it is known that $[1$, p. 277]

$$
\left|s_{n}^{(k)}(\theta)\right|<A_{k} n^{1 / 2-k} \theta^{-1-k}(\sin \theta)^{-1 / 2} .
$$

Also [1, p. 274]

$$
\left|s_{n}^{(1)}(\theta)\right| \leqq 2 \quad(\pi / 2 \leqq \theta \leqq \pi) .
$$

By (10), the $(C, k)$-means of $\sum_{0}^{\infty} Y_{n}(P)$ are equal to

$$
\sigma_{n}^{(k)}(P)=\frac{1}{4 \pi} \int_{E} s_{n}^{(k)}(P Q) d \lambda(Q) .
$$

For $P$ in $S-E$, let $E^{*}$ be the set of all $Q$ in $E$ such that $P Q<\pi$. Since every single point has $\lambda$-measure zero, we may replace $E$ by $E^{*}$ in (13); taking $k=1,(11)$ and (12) show that $s_{n}^{(1)}(P Q) \rightarrow 0$ boundedly as $n \rightarrow \infty$, for $Q$ in $E^{*}$. Consequently $\sigma_{n}^{(1)}(P) \rightarrow 0$, and part (i) of the theorem follows.

Part (iii) is proved similarly if we note that for some $\delta>0$ we have $\delta \leqq P Q \leqq \pi-\delta$ for every $Q$ in $E$, and apply (11).

We conclude with an analogue of a theorem of Salem and Zygmund $[4$, p. 25$]$; compare also $[5$, p. 584$]$. 
THEOREM C. If $E$ is a closed subset of $S$, the following statements are equivalent:

(a) The capacity of $E$ is positive.

(b) There exists a measure $\lambda$, concentrated on $E$ and not identically zero, whose Laplace-Stieltjes series is $\sum_{0}^{\infty} Y_{n}(P)$, such that

$$
-\sum_{1}^{\infty} Y_{n}(P) / n(n+1)
$$

is the Laplace series of a continuous function.

(c) Same as (b), with "continuous" replaced by "bounded."

Proof. We have proved above that (a) implies (b), and (b) obviously implies (c). If (c) holds and $Y_{n}(P)$ is given by (10) then the function $F$ whose Laplace series is $-\sum_{1}^{\infty} Y_{n}(P) / n(n+1)$ is given by (5). Since $F$ is bounded, harmonic, and not constant in $S-E$, the capacity of $E$ cannot be zero. Hence (c) implies (a).

\section{REFERENCES}

1. Leopold Fejér, Über die Summierbarkeit der Laplaceschen Reihe durch arithmetische Mittel, Math. Zeit. vol. 24 (1926) pp. 267-284.

2. Walter Rudin, Integral representation of continuous functions, Trans. Amer. Math. Soc. vol. 68 (1950) pp. 279-286.

3. ㄴ, Uniqueness theory for Laplace series, Trans. Amer. Math. Soc. vol. 68 (1950) pp. 287-303.

4. Raphael Salem and Antoni Zygmund, Capacity of sets and Fourier series, Trans. Amer. Math. Soc. vol. 59 (1946) pp. 23-41.

5. V. L. Shapiro, Logarithmic capacity of sets and double trigonometric series, Canadian Journal of Mathematics vol. 6 (1954) pp. 582-592.

6. Ch. J. de la Vallée-Poussin, Le potentiel logarithmique, Paris, 1949.

UNIVERSITY OF ROCHESTER 